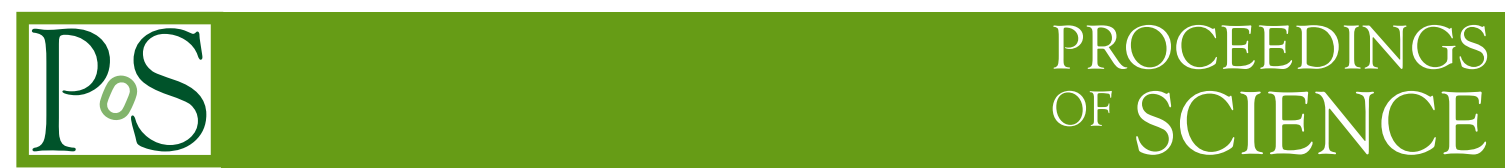

\title{
Fermi-LAT Recent Results on Gamma-Ray Bursts
}

\author{
Frédéric Piron*, on behalf of the Fermi LAT and GBM Collaborations \\ Laboratoire Univers et Particules de Montpellier, Université Montpellier 2, CNRS/IN2P3, \\ Montpellier, France \\ pironein2p3.fr
}

The Fermi Large Area Telescope (LAT; [8]) is a pair-conversion detector of high-energy gamma rays of energies ranging from $20 \mathrm{MeV}$ to more than $300 \mathrm{GeV}$. Since the launch in June 2008, it has been operating in synergy with the Gamma-ray Burst Monitor (GBM; [13]), which covers the entire unocculted sky and is designed for gamma-ray transients' detection and spectroscopy between $8 \mathrm{keV}$ and $40 \mathrm{MeV}$. The LAT detects $\sim 9$ gamma-ray bursts (GRB) per year, which represents $\sim 8 \%$ of the GBM bursts occuring in its field of view. We present a systematic study of the GRB properties observed with the LAT, and discuss the LAT non-detections of some GBM bright and hard bursts, in an effort to understand the difference in GRB rates from both instruments.

Gamma-Ray Bursts 2012 Conference-GRB2012,

May 07-11, 2012

Munich, Germany

${ }^{*}$ Speaker. 

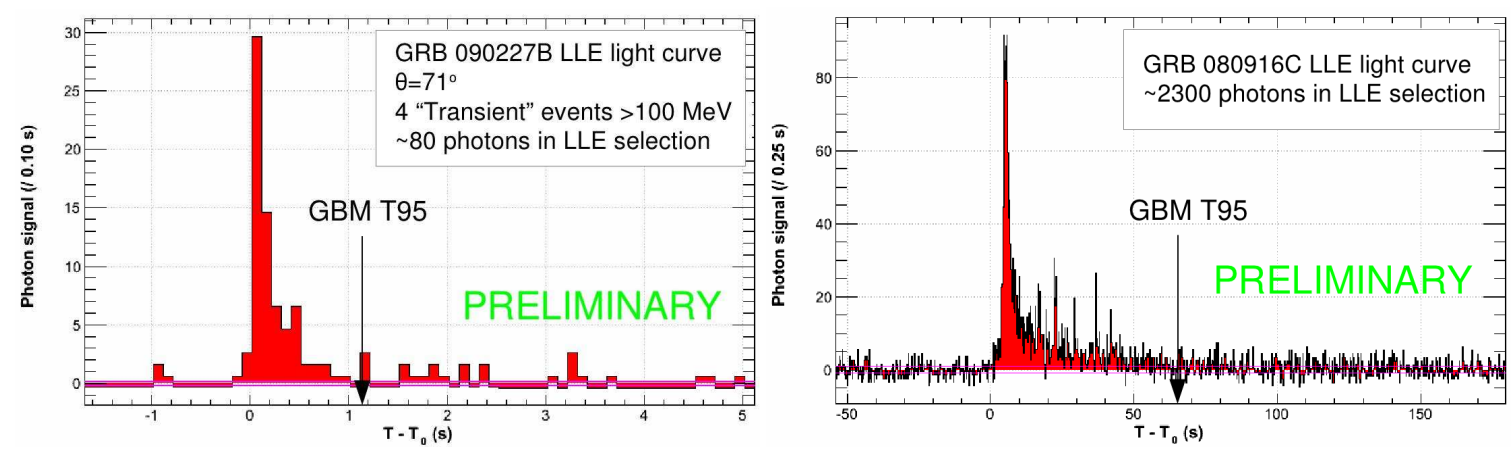

Figure 1: LAT Low-Energy (LLE) light curves of GRB 090227B (left) and GRB 080916C (right).

\section{Introduction}

The most important observations of GRBs performed by the LAT over the past $\sim 1.5$ year are presented in dedicated contributions at this conference, in particular the detailed studies of the photospheric emission from GRB 110721A [15] and of the afterglow emission from GRB 110731A [10]. In addition, theoretical aspects of GRB emission are thoroughly discussed in other review presentations. In this contribution, we therefore cover the LAT observational results, focusing on recent populations studies with the LAT and on GRB common properties at high energies. In section 2, we present what has been seen with the LAT in 3 years of operations, highlighting results from the first LAT GRB catalog. In section 3, we discuss the LAT GRB rate and examine the LAT non-detections of GBM bright GRBs. Whereas about half of the GBM GRBs occur in the LAT field of view (FoV), we investigated why only $\sim 8 \%$ are detected with the LAT above $100 \mathrm{MeV}$.

\section{The First LAT Gamma-Ray Burst Catalog}

The first LAT GRB catalog is a systematic study of GRBs at high energies $(\gtrsim 20 \mathrm{MeV})$, covering a 3-year period starting from the beginning of the nominal Fermi science operations in August 2008. It aims at characterizing GRB temporal and spectral properties at high energies, including tabulated GRB parameters along with details on the analysis methodology and tools and their caveats. In the following, we describe some of the ingredients necessary to this work and present selected results.

\subsection{Ingredients for GRB Analysis with the LAT}

Fermi was designed with the capability to repoint in the direction of a bright GRB and keep its position near the centre of the LAT FoV for several hours, subject to Earth-limb constraints. This repointing occurs autonomously in response to requests from the GBM or the LAT, with adjustable brightness thresholds. Each Autonomous Repoint Request (ARR) causes rapid variations of the source off-axis angle in the LAT FoV, making the estimate of the background quite challenging ${ }^{1}$. In particular, the usual background-estimation method which consists of extrapolating fits of the event

\footnotetext{
${ }^{1}$ Another consequence is the huge variation of the LAT exposure, which strongly modulates the observed light curves in detected count space.
} 

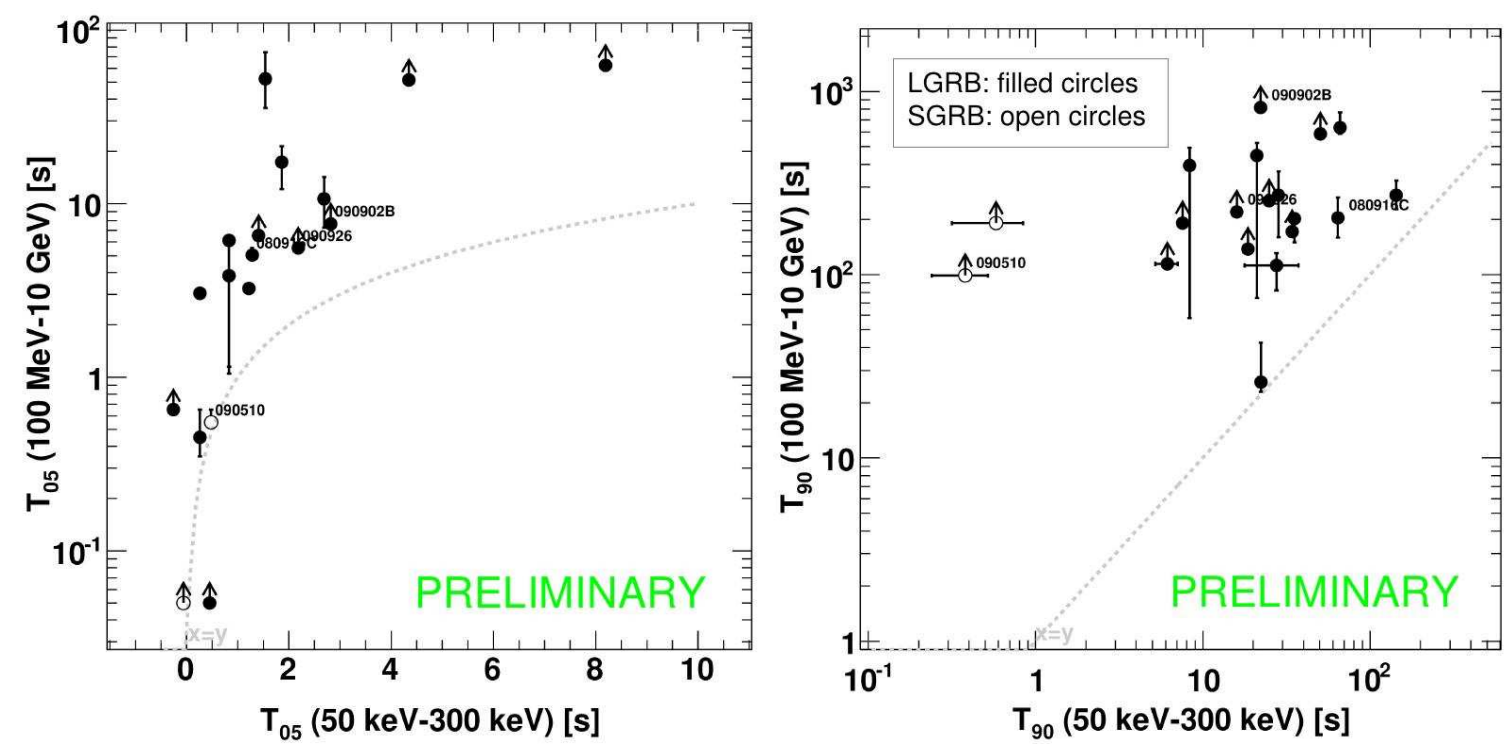

Figure 2: Onset time ( $\mathrm{T}_{05}$, left) and duration ( $\mathrm{T}_{90}$, right) of the GRB emission observed by the GBM (50-300 $\mathrm{keV})$ and the $\mathrm{LAT}(>100 \mathrm{MeV})$.

rate before the burst (or interpolating from fits to pre- and post-burst time intervals) provides wrong estimates in the case of ARRs. For the analyses included in the LAT GRB catalog, we instead make use of the background-estimation tool developed by the LAT collaboration and described in detail in [1]. This tool works in any observational conditions and provides estimates with $10-15 \%$ accuracy.

Another important ingredient for GRB studies is the use of the "LAT Low-Energy" (LLE) event class. In the catalog work, most GRBs are detected by performing an unbinned likelihood analysis based on the standard event selection (the so-called "transient"-class events) above 100 $\mathrm{MeV}$. However, some GRBs are too weak or at too high off-axis angles $\left(\gtrsim 70^{\circ}\right)$ to be significantly detected. In order to recover significant signal in these situations, we introduced the LLE event class, which corresponds to relaxed event selection criteria. It provides significantly higher effective area at tens-of-MeV energies and at larger off-axis angles (Fig. 1, left) and, for the bright GRBs, the needed statistics to study the temporal properties at low energies in the LAT (Fig. 1, right).

\subsection{LAT GRB Detections and Durations}

Whereas the GBM detects 250 GRBs per year [14] (half of them occuring in the LAT FoV), the LAT detected 35 GRBs in 3 years of operations. This includes 30 long and 5 short GRBs, and 7 "LLE-only" GRBs. Approximately half of these detections benefited from more accurate followup localizations by Swift and ground-based observatories. To date, 9 redshift measurements are available for LAT GRBs, ranging from $\mathrm{z}=0.74$ (GRB 090328) to $\mathrm{z}=4.35$ (GRB 080916C).

The GRB emission detected by the LAT above $100 \mathrm{MeV}$ is systematically delayed with respect to the emission observed with the GBM at hundreds-of-keV energies (Fig. 2, left). It also lasts sys- 

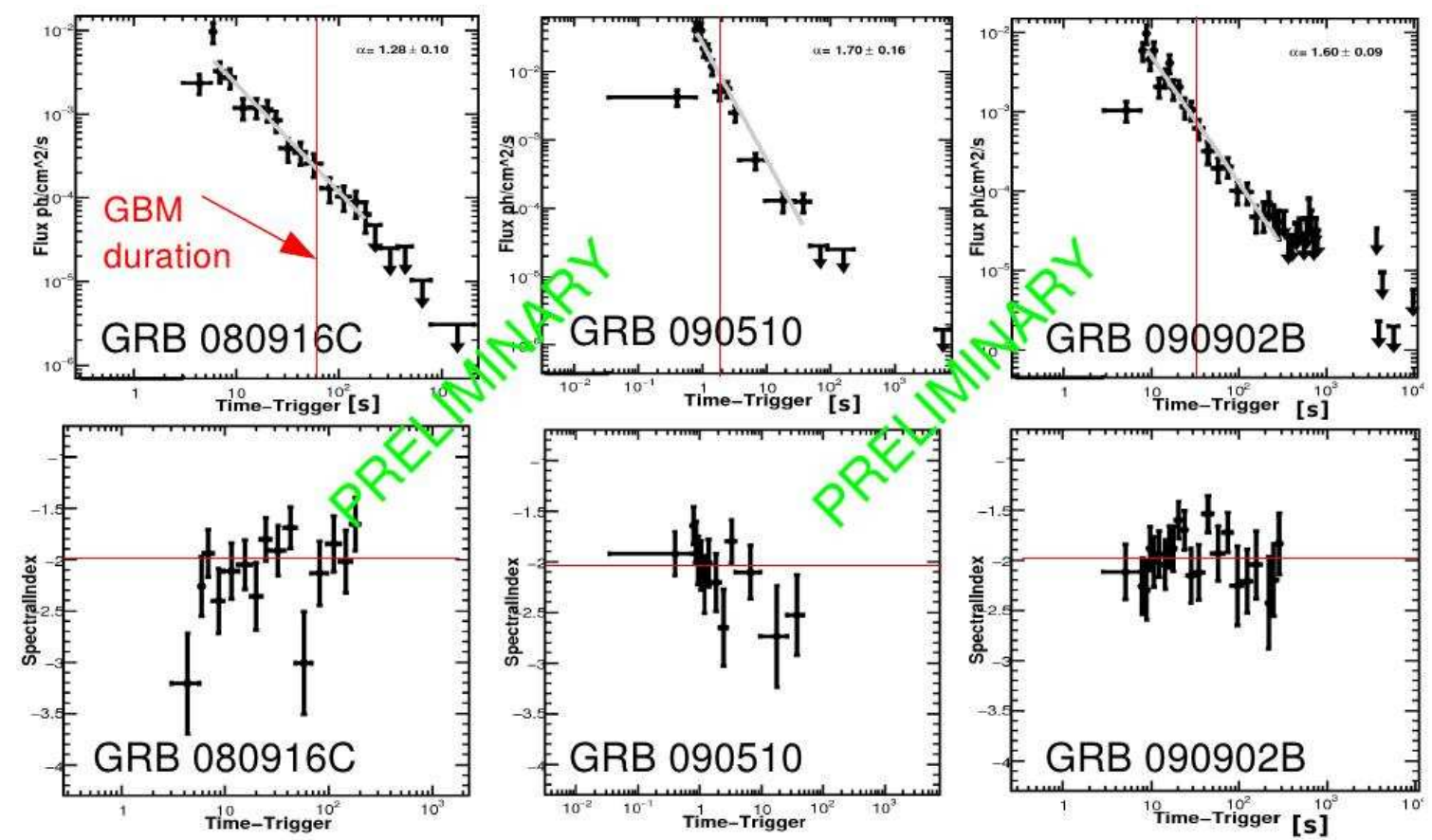

Figure 3: Integrated flux above $100 \mathrm{MeV}$ (top row) and spectral index (bottom row) as a function of time for 3 LAT bright GRBs.

tematically longer (Fig. 2, right), which could be interpreted as different origins of both emissions ${ }^{2}$ or by a relatively better sensitivity of the LAT, as the GBM is a background-dominated detector. The origin of the high-energy emission seen in the LAT is much less unclear at later times, as the LAT flux above $100 \mathrm{MeV}$ decays as a power-law in time (Fig. 3), with decay indices between -1.8 and -0.8 which are consistent with an afterglow interpretation. In particular, no obvious pattern is seen in the spectral evolution, and the photon spectral indices typically average around -2 .

\subsection{Fluences and Energetics}

Not surprisingly, the GRBs detected by the LAT are found to be among the GBM brightest $\mathrm{GRBs}^{3}$ (Fig. 4, left). In addition, remarkable properties of GRBs were discovered with the LAT at high energies. Firstly, whereas the fluence in the LAT energy range is $\approx 10 \%$ of the fluence in the GBM energy range for long GRBs, short GRBs have a larger fluence ratio (Fig. 4, right). Although this result certainly requires more GRB statistics to be firmly confirmed, it already suggests different energy outputs above $100 \mathrm{MeV}$ from GRBs depending on their progenitors. Secondly, some evidence of a class of hyper-energetic GRBs was found, with four exceptionally bright events (GRBs 080916C, 090510, 090902B and 090926A) [2, 6, 3, 7]. This important result can not be simply explained by the proximity of these GRBs, as their redshifts are distributed from 0.90 to 4.35 .

\footnotetext{
${ }^{2}$ However, the very fast variability seen in the right panel of Fig. 1, seems to favor an internal shock origin of the high-energy emission.

${ }^{3}$ Note that selection effects due to ARRs should be investigated though.
} 

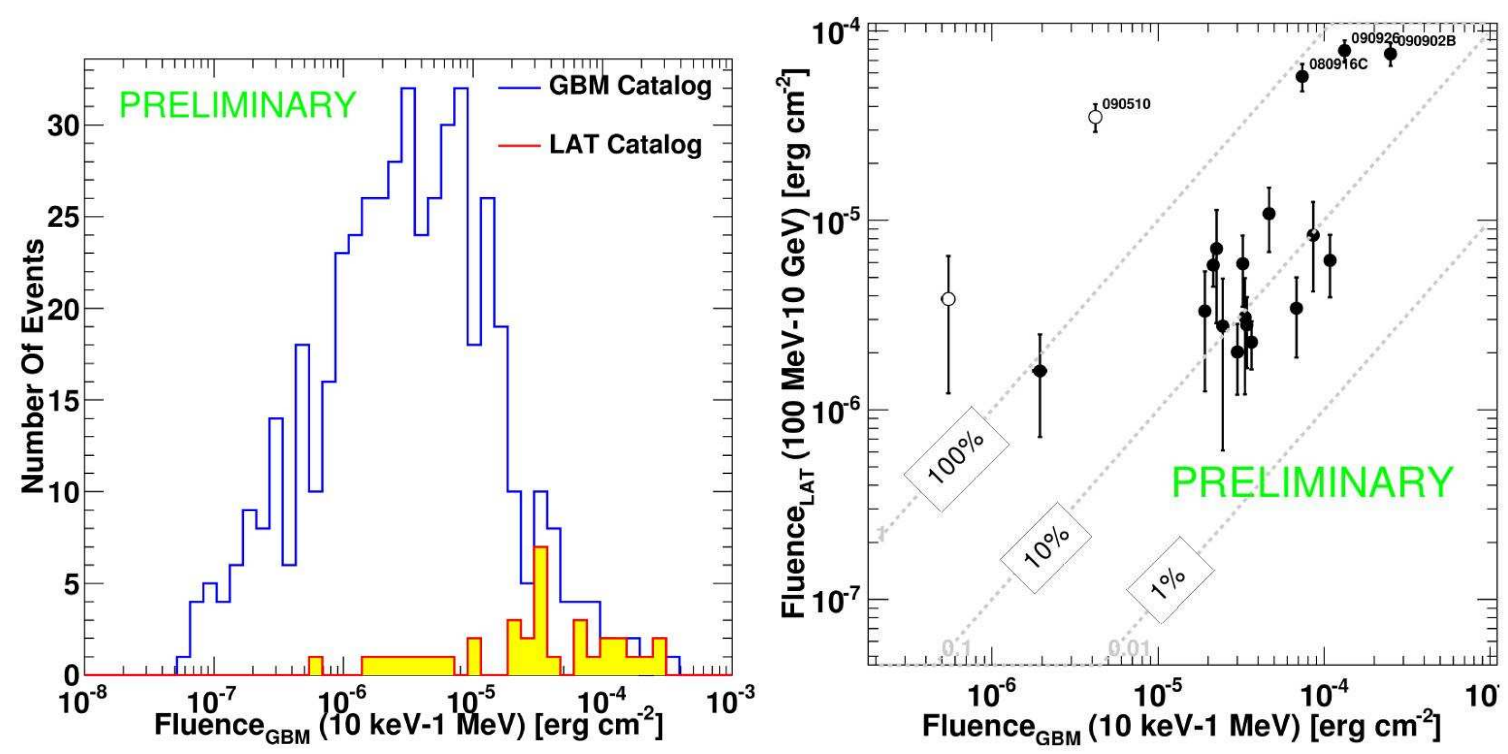

Figure 4: (Left) Fluence in the GBM energy range and within the GBM $\mathrm{T}_{90}$, for the entire sample in the GBM spectral catalog [12] and for the LAT GRBs only. (Right) Comparison of the LAT GRB fluences, computed in the GBM and LAT respective energy ranges and T90's. Filled circles indicate long GRBs, and open circles short GRBs.

Fig. 5 (left) shows that the LAT GRBs are among the most energetic GRBs (GRB 090510 being the most energetic short one), with no particular trend in redshift with respect to other GRB samples (GBM, Swift). The right panel in the same figure shows that the rest-frame emission from these GRBs can frequently reach several tens-of-GeV energies, which is a very good sign for the detection prospects of future very high-energy observatories such as the High Altitude Water Cherenkov experiment [16] and the Cherenkov Telescope Array. These high-energy photons were extensively used to set different kinds of constraints. First of all, they provided lower limits on the mean Lorentz factor $\Gamma$ of the GRB jets through compacity arguments, and helped to demonstrate that both long and short GRBs can have high outflow rapidity [2, 6, 3, 7], a key result for GRB modelling (see also section 3). Secondly, high-energy gamma rays can be absorbed by the Extragalatic Background Light (EBL) when travelling from the emitting region to the observer. Only photons with energies above $\sim 10 \mathrm{GeV}$ suffer from pair creation on the EBL, and they can be used to probe this cosmic diffuse radiation field as a function of redshift in the optical-UV range [5]. Finally, the LAT observations of $>10 \mathrm{GeV}$ photons from bright GRBs also provided the best lower limits on the energy scale at which postulated quantum-gravity effects create violations of Lorentz invariance. These constraints strongly disfavour models which predict a linear variation of the speed of light with photon energy below the Planck energy scale (i.e., $<1.22 \times 10^{19} \mathrm{GeV}$ ) [4].

\section{Non-Detections of GBM Bright Gamma-Ray Bursts}

Whereas $\sim 9.3 \mathrm{GRBs}$ with more than 10 photons above $100 \mathrm{MeV}$ were expected per year from pre-launch estimates [9], a mean LAT GRB rate of $\sim 6.3$ GRBs per year is obtained from the first LAT GRB catalog, based on the number of photons predicted by the likelihood fits within the LAT 

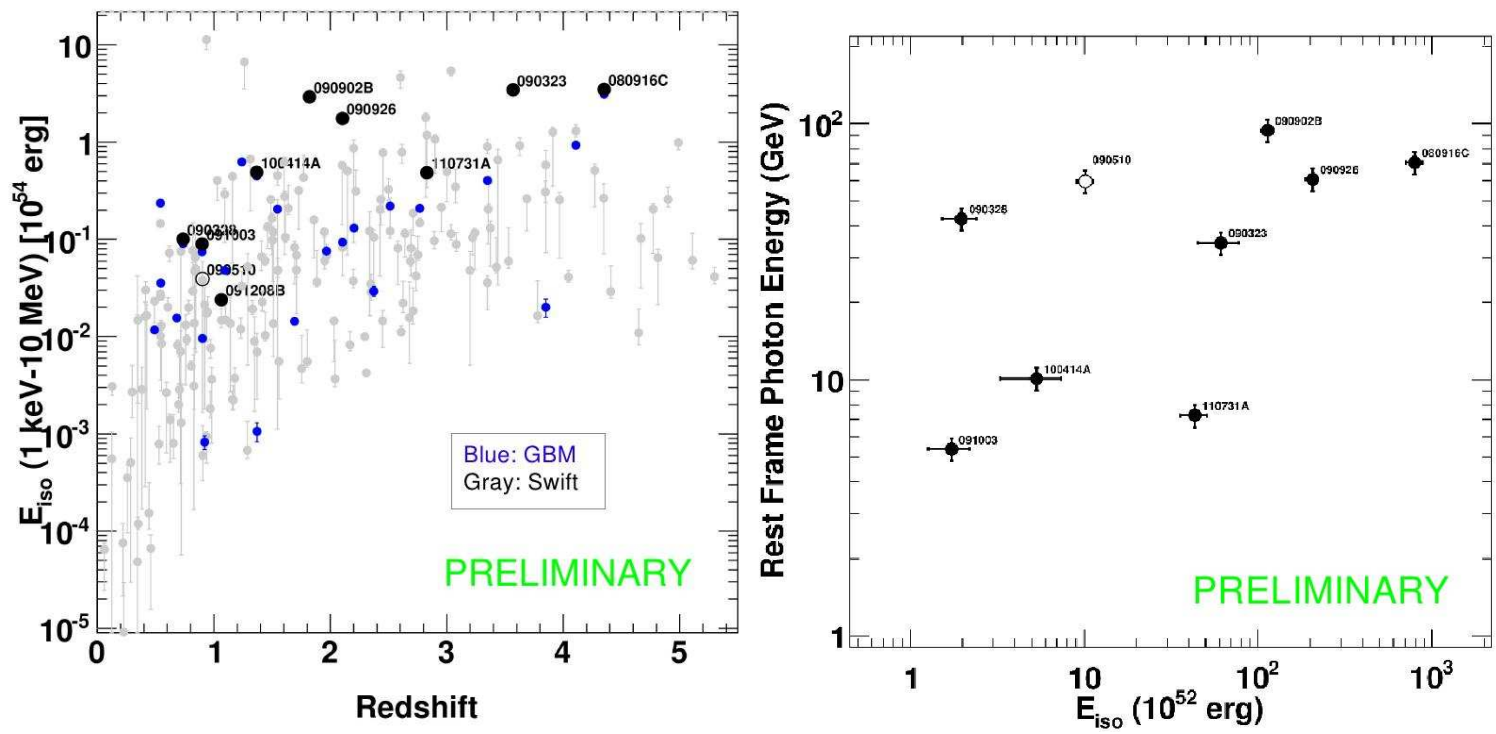

Figure 5: (Left) Isotropic equivalent energy from $1 \mathrm{keV}$ to $10 \mathrm{MeV}$ during the GBM $\mathrm{T}_{90}$. We compare the 9 LAT GRBs with measured redshifts with the samples of GBM [12] and Swift [11] GRBs. (Right) Rest-frame energy of the highest-energy photons from the GRBs detected by the LAT.

$\mathrm{T}_{90}$. In spite of different systematic uncertainties arising in these analyses ${ }^{4}$, this lack of LAT GRB detections suggests that the GRB emission is not as prominent or strong in the LAT energy range. It raises the question whether the high-energy emission is suppressed and if spectral cutoffs are more common than anticipated, similarly to the attenuated spectrum of GRB 090926A [7].

In order to investigate this question, we estimated the fraction of GBM GRBs that should have been detected by the LAT. Firstly, we examined a sample of $30 \mathrm{GBM}$ bright and hard GRBs, with $>70$ counts/s in the BGO detectors and with a good measurement of the high-energy slope $\beta$ of the Band spectrum $(\Delta \beta<0.5)$. Comparing upper limits on the LAT flux $(0.1-10 \mathrm{GeV})$ over the GBM $\mathrm{T}_{90}$ with predictions from the extrapolation of the spectral fits to GBM data, we found that $\sim 50 \%$ (15 GRBs) should have been detected by the LAT. In a second step, we included the LAT data ("transient"-class events) above $100 \mathrm{MeV}$ in the spectral fits, which yielded considerably softer $\beta$ values (Fig. 6, left) and decreased this fraction down to $\sim 23 \%$. Finally, we repeated the GBM-LAT joint fits, adding a spectal softening in the model between the GBM/BGO and the LAT energy ranges. This modification to the spectrum improved the fits significantly for $20 \%$ of the GRBs (6 out of 30), implying that a degree of spectral softening is required to explain their LAT non-detection. The rest $80 \%$ of the GRBs remained statistically consistent with a softer $\beta$. It is interesting to note that these 6 GRBs have the smallest $\Delta \beta$ values (and no other particular characteristic), indicating that only a very accurate spectroscopy is sensitive to this spectral feature. Assuming that the softening is due to internal opacity effects, we finally set upper limits on the jet mean Lorentz factors of the $6 \mathrm{GRBs}$, assuming a (conservative) $100 \mathrm{~ms}$ variability time scale. The comparison of these limits to other estimates obtained for the 4 LAT brightest GRBs (Fig. 6, right)

\footnotetext{
${ }^{4}$ For instance, the extrapolations from the BATSE energy range to the LAT energy range as performed in [9] are uncertain due to the large lever arm and to errors on the high-energy spectral slope $\beta$. Moreover, these past estimates used simple detection thresholds and idealized backgrounds.
} 

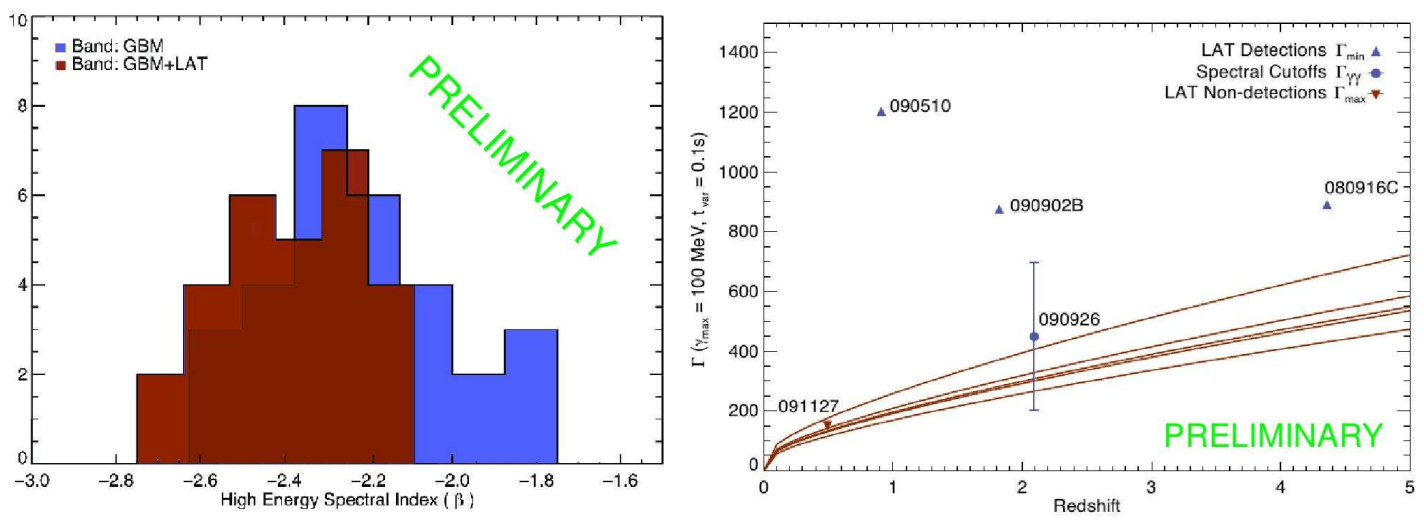

Figure 6: (Left) Distribution of the high-energy spectral slope $\beta$ for the GBM sample of bright GRBs (see text), from fits to the GBM-only data or from GBM-LAT joint fits. (Right) Upper limits $\Gamma_{\max }$ on the jet mean Lorentz factors for the 6 GRBs exhibiting a spectral softening. We only know the redshift for GRB 091127 (brown triangle), so we set $\Gamma_{\max }(z)$ for the rest: GRBs 080925, 081207, 090131, 090528B, and 100724B (brown curves). Lower limits $\Gamma_{\min }$ (GRBs 080916C, 090510, 090902B) and measurements (GRB 090926A) for LAT bright GRBs are superimposed (blue triangles and point). The target photon field for $\gamma \gamma$ absorption is assumed uniform, isotropic and time-independent, but the error bar for GRB 090926A accounts for different models, illustrating the overall scaling that could be applied to the entire figure.

indicates a relatively broad range of possible $\Gamma$ values among Fermi GRBs.

\section{Conclusions and Perspective}

The GBM and LAT instruments onboard the Fermi space observatory have jointly detected the keV-MeV-GeV emission from a large sample of GRBs. The LAT detected 35 GRBs in the first 3 years of Fermi operations, by means of the standard likelihood technique above $100 \mathrm{MeV}$ and/or using the new LAT Low-Energy (LLE) event class. A population study indicates (or confirms) interesting patterns, like the delayed onset and the temporal extension of the GRB emission observed in the LAT above $100 \mathrm{MeV}$ with respect to the emission detected by the GBM at lower energies. Emergent groups are also observed: LAT GRBs are among the brightest and most energetic GBM GRBs, and the evidence of a class of hyper-energetic GRBs is growing. The first LAT GRB catalog will certainly be a valuable tool for future theoretical research and a useful informational resource for scientists who wish to analyze LAT GRB data.

Fewer GRBs are detected by the LAT than would be expected by extrapolating BATSE or GBM spectra. The need for an overall softer spectrum or for high-energy cutoffs in GRB spectra at $\mathrm{MeV}$ energies is confirmed ( $20 \%$ in the sample examined). Assuming that this spectral softening arises from internal opacity effects, the range of values for the mean Lorentz factors $\Gamma$ of Fermi GRB jets is found to be broader than those derived only from the LAT bright GRBs.

More LAT GRBs will certainly help to understand these spectral properties and to shed light on the LAT GRB rate in the future. The LLE data, which fill the gap between the GBM and the LAT energy ranges, could also help to detect more cutoffs which are not detectable with the standard analysis of LAT data. Whereas the standard "transient" event selection runs out of effective area below $\sim 100 \mathrm{MeV}$, the LLE event selection provides plenty of statistics to probe GRB spectral 
cutoffs in the tens-of-MeV-energy region. The validation of the spectral reconstruction technique with LLE data is ongoing. Systematic uncertainties in LLE effective area and point-spread-function are being investigated in various configurations (event energies, source off-axis angles, background levels), and the first validation results are encouraging as shown at the conference. In parallel, the preparation for the public release of LLE data at the Fermi Science Support Center ${ }^{5}$ has started. In the near future, any scientist interested in the LAT observations of GRBs will be able to access the LLE data and the instrument responses for any past or new LLE-detected GRB.

\section{References}

[1] Abdo, A. A., et al., Fermi Observations of High-Energy Gamma-Ray Emission from GRB 080825C, ApJ, 707, 580 (2009a)

[2] Abdo, A. A., et al., Fermi Observations of High-Energy Gamma-Ray Emission from GRB 080916C, Science, 323, 1688 (2009b)

[3] Abdo, A. A., et al., Fermi Observations of GRB 090902B: a Distinct Spectral Component in the Prompt and Delayed Emission, ApJ Letters, 706, 138 (2009c)

[4] Abdo, A. A., et al., A limit on the Variation of the Speed of Light arising from Quantum Gravity Effects, Nature, 462, 331 (2009d)

[5] Abdo, A. A., et al., Fermi Large Area Telescope Constraints on the Gamma-Ray Opacity of the Universe, ApJ, 723, 1082 (2010)

[6] Ackerman, M., et al., Fermi Observations of GRB 090510: a Short-Hard Gamma-Ray Burst with an Additional Hard Power-Law Component from $10 \mathrm{keV}$ to GeV Energies, ApJ, 716, 1178 (2010a)

[7] Ackerman, M., et al., Detection of a Spectral Break in the Extra Hard Component of GRB 090926A, ApJ, 729, 114 (2010b)

[8] Atwood, W. B., et al., The Large Area Telescope on the Fermi Gamma-Ray Space Telescope Mission, ApJ, 697, 1071 (2009)

[9] Band, D. L., et al., Prospects for GRB Science with the Fermi Large Area Telescope, ApJ, 701, 1673 (2009)

[10] Bregeon, J., Broadband Observations of GRB 110731A with Fermi, Swift, GROND and MOA, in proceedings of “Gamma-Ray Bursts 2012 Conference”, PoS(GRB2012)020

[11] Butler, N. R., et al., A Complete Catalog of Swift Gamma-Ray Burst Spectra and Durations: Demise of a Physical Origin for Pre-Swift High-Energy Correlations, ApJ, 671, 656 (2007)

[12] Goldstein, A., et al., The Fermi GBM Gamma-Ray Burst Spectral Catalog: The First Two Years, ApJSS, 199, 19 (2012)

[13] Meegan, C., et al., The Fermi Gamma-Ray Burst Monitor, ApJ, 702, 791 (2009)

[14] Paciesas, W. S., et al., The Fermi GBM Gamma-Ray Burst Catalog: The First Two Years, ApJSS, 199, 18 (2012)

[15] Ryde, F., The Photosphere in Gamma-Ray Bursts: Lessons Learned from Fermi, in proceedings of "Gamma-Ray Bursts 2012 Conference", PoS(GRB2012)011

[16] Zabarov, D., The HAWC experiment and its sensitivity to gamma-ray bursts, in proceedings of "Gamma-Ray Bursts 2012 Conference", PoS(GRB2012)122

\footnotetext{
${ }^{5}$ http: //fermi.gsfc.nasa.gov/
} 